\title{
Guidelines for medical management of hyperglycaemia in type 2 diabetes and duality of interest
}

\author{
D. L. Eizirik
}

Received: 31 October 2008 / Accepted: 4 November 2008 / Published online: 26 November 2008

(C) Springer-Verlag 2008

To the Editor: I read with great interest the Consensus Statement article by Nathan et al. [1] on behalf of the American Diabetes Association (ADA) and the European Association for the Study of Diabetes (EASD), suggesting a consensus algorithm for initiation and adjustment of therapy for the medical management of hyperglycaemia in type 2 diabetes. In describing the process used to develop the algorithm, the authors mentioned two sources: (1) clinical trials that address the effectiveness and safety of different modalities of therapy; and (2) clinical judgement, i.e., the collective knowledge and clinical experience of the authors, which takes into account benefits, risks and costs in the treatment of diabetes [1]. Since the authors point to the "paucity of high-quality evidence in the form of wellcontrolled clinical trials that directly compare different diabetes treatment regimens', they propose that the scarceevidence-based information should be supplemented by 'value judgements, where the benefits of treatment are weighed against risks and costs in a subjective fashion' [1]. This need for value judgement, in particular in the context of the preparation of a consensus statement that may influence the therapy of millions of patients, presupposes a high level of independence and balanced judgement from the authors. The carefully written and critical description of the different modalities of treatment suggests that this was probably the case for the present guidelines [1].

At the end of the article there is a lengthy description of dualities of interest, ranging from two to 13 reported dualities

\footnotetext{
D. L. Eizirik $(\bowtie)$

Laboratory of Experimental Medicine,

Université Libre de Bruxelles (ULB),

808 Route de Lennik,

Brussels 1070, Belgium

e-mail: deizirik@ulb.ac.be
}

per author (mean $\pm \mathrm{SEM}, 10.1 \pm 1.4$ ), most of them related to pharmaceutical companies involved in the production and commercialisation of many of the agents described and recommended in the guidelines. As defined by The Endocrine Society, a duality of interest is present when two or more interests are potentially in conflict, while a conflict of interest exists when a given relationship or practice gives rise to two or more contradictory interests [2]. Specifically, Thompson describes conflict of interest as 'a set of conditions in which professional judgement concerning a primary interest (such as patient welfare or the validity of research) tends to be unduly influenced by a secondary interest (such as financial gain)' [3]. The dualities of interest listed for authors of the present guidelines include research grants, serving on scientific advisory boards and receipt of honoraria for speaking engagements [1]. In other words, part of the research funding and private income of several of the authors of the present guidelines may depend on pharmaceutical companies that benefit from the incorporation of their drugs in the guidelines. This gives rise to duality of interest and, potentially, to conflict of interest.

Is this a source of real concern? On the 'no' side we can count on the high professional standing of the authors and on the hypothetical if unlikely possibility that such a large number of dualities of interest (five of the seven authors report more than ten dualities of interest) may somehow counterbalance each other, leaving the author(s) in a relatively balanced position. On the 'yes' side is the accumulating evidence that dualities of interest do indeed change conclusions and interpretation of studies. For instance, a broad review (based on 1,140 studies) evaluating the relationship between industry sponsorship and outcome in original research indicates a statistically significant association between industry sponsorship and pro-industry conclusions (pooled Mantel-Haenszel odds ratio 3.60) [4]. Moreover, 
there is evidence that acceptance of gifts by physicians increases the possibility that they will prescribe the drugs made by the pharmaceutical company donors, independently of the scientific data supporting these clinical decisions [5]. Finally, expert clinicians advising pharmaceutical companies gain access to privileged and non-peer reviewed information, and work with highly committed individuals whose professional lives revolve around a single product, generating another potential source of bias [6].

How can we solve this conundrum? One possibility is to restrict the preparation of consensus statements to colleagues with few or, ideally, no dualities of interest with pharmaceutical companies that may benefit from the guidelines (these colleagues do exist). Another alternative is to invite one or two independent commentator(s), with no relevant duality of interest, to analyse the guidelines and provide her/his views to be published as an addendum to the text. Additional alternatives may be considered; the crucial point is to acknowledge that duality of interest is a serious issue that must be carefully considered and addressed by learned medical societies and healthcare agencies.
Duality of interest The author declares that there is no duality of interest associated with this manuscript.

\section{References}

1. Nathan DM, Buse JB, Davidson MB et al (2008) Medical management of hyperglycaemia in type 2 diabetes mellitus: a consensus algorithm for the initiation and adjustment of therapy. A consensus statement from the American Diabetes Association and the European Association for the Study of Diabetes. Diabetologia doi:10.1007/s00125-008-1157-y

2. Komesaroff PA, Bach MA, Danoff A et al (2004) The Endocrine Society Ethics Advisory Committee: ethical aspects of conflicts of interest, October 2003. Endocrinology 145:3032-3041

3. Thomson DF (1993) Understanding financial conflicts of interest. N Engl J Med 329:573-576

4. Bekelman JE, Li Y, Gross CP (2003) Scope and impact of financial conflicts of interest in biomedical research: a systematic review. JAMA 289:454-465

5. Komesaroff PA (2005) Ethical issues in the relationships with industry: An ongoing challenge. New guidelines open for public comment. J Paediatr Child Health 41:558-560

6. Gale EAM (2003) Between two cultures: the expert clinician and the pharmaceutical industry. Clin Med 3:538-541 